hematopoiesis. First, it may be possible to induce human HSC expansion in vitro or in vivo via pharmacologic activation of NRF2. If successful, such capability could have a significant impact on the field of HSC transplantation. Second, pharmacologic induction of NRF2 could serve to mitigate the potentially fatal effects of radiation-induced hematopoietic failure in victims of acute radiation sickness. This latter possibility has major public health implications, given the ongoing worldwide threat of radiological or improvised nuclear devices as weapons of terrorism (15). Commensurate with this, the development of effective therapies to mitigate acute radiation sickness is a high priority for the US federal government (16).

In summary, the study by Kim et al. (13) provides new insights into the fundamental role of NRF2 in regulating the hematopoietic response to ionizing radiation. Of equal importance, NRF2 represents a new mechanistic target for the development of therapeutics to accelerate hematopoietic reconstitution both in victims of acute radiation sickness and patients undergoing stem cell transplantation.

Address correspondence to: John P. Chute, Division of Hematology/Oncology, Room 545, Orthopedic Hospital Research Center, UCLA, Los Angeles, California 90095, USA. Phone: 310.206.3037; E-mail: JChute@mednet.ucla.edu.

\footnotetext{
1. Till J, McCulloch E. A direct measurement of the radiation sensitivity of normal mouse bone marrow cells. Radiat Res. 2012;178(2):AV3-AV7.

2. McCulloch E, Till J. The radiation sensitivity of normal mouse bone marrow cells, determined by quantitative marrow transplantation into irradiated mice. Radiat Res. 1960;13:115-125.

3. Okita T. Review of thirty years study of Hiroshima and Nagasaki atomic bomb survivors. II. Biological effects. A. Acute effects. J Radiat Res. 1975;16(suppl):49-66.

4. Hiroshima Day Committee. Hiroshima and Nagasaki Bombing: Facts about the atomic bomb. Hiroshima Day Committee Web site. http://www. hiroshimacommittee.org/Facts_NagasakiAndHiro shimaBombing.htm. Accessed January 24, 2014.

5. Till J, McCulloch E, Siminovitch L. A stochastic model of stem cell proliferation, based on the growth of spleen colony forming cells. Proc Natl Acad Sci U S A. 1964;51:29-36.

6 . Snell GD. Methods for the study of histocompatibility genes. J Genet. 1948;49(2):87-108.

7. Thomas ED, Lochte HL Jr, Ferrebee JW. Irradiation
}

of the entire body and marrow transplantation: some observations and comments. Blood. 1959;14(1):1-23.

8. Farese AM, et al. Filgrastim improves survival in lethally irradiated nonhuman primates. Radiat Res. 2013;179(1):89-100.

9. Guinan E, et al. Bactericidal/permeability-increasing protein (rBPI21) and fluoroquinolone mitigate radiation-induced bone marrow aplasia and death. Sci Transl Med. 2011;3(110):110ra-118.

10. Burdelya L, et al. An agonist of toll-like receptor 5 has radioprotective activity in mouse and primate models. Science. 2008;320(5873):226-230.

11. Himburg $\mathrm{H}$, et al. Pleiotrophin regulates the expansion and regeneration of hematopoietic stem cells. Nat Med. 2010;16(4):475-482.

12. Doan PL, et al. Epidermal growth factor regulates hematopoietic regeneration after radiation injury. Nat Med. 2013;19(3):295-304.

13. Kim J-H, et al. NRF2-mediated Notch pathway activation enhances hematopoietic reconstitution following myelosuppressive radiation. J Clin Invest. 2014;124(2):730-741

14. Kensler TW, Wakabayashi N, Biswal S. Cell survival responses to environmental stresses via the Keap1Nrf2-ARE pathway. Annu Rev Pharmacol Toxicol. 2007;47:89-116.

15. Kim SB, et al. Targeting of Nrf2 induces DNA damage signaling and protects colonic epithelial cells from ionizing radiation. Proc Natl Acad Sci U S A. 2012;109(43):E2949-E2955.

16. Weinstock DM, et al. Radiologic and nuclear events: contingency planning for hematologists/ oncologists. Blood. 2008;111(12):5440-5445.

17. Hafer N, et al. NIAID/NIH radiation/nuclear countermeasures product research and development program. Health Phys. 2010;98(6):903-905.

\title{
Fanning the flames to regenerate the heart
}

\author{
Paul R. Riley
}

Department of Physiology, Anatomy and Genetics, University of Oxford, Oxford, United Kingdom.

\begin{abstract}
Damage to the adult mammalian heart is irreversible, and lost cells are not replaced through regeneration. In neonatal mice, prior to $\mathrm{P} 7$, heart tissue can be regenerated after injury; however, the factors that facilitate cardiac regeneration in the neonatal heart are not known. In this issue of the JCI, Aurora and colleagues evaluated the immune response following myocardial infarction in P1 mice compared with that in P14 mice, which have lost their regenerative capacity, and identified a population of macrophages as mediators of cardiac repair. Further understanding of the immune modulators that promote the regenerative properties of this macrophage subset could potentially be exploited to recapitulate regenerative function in the adult heart.
\end{abstract}

\section{Repair without regeneration in the damaged adult heart}

Myocardial infarction (MI), commonly known as heart attack, results in the loss of around 1 billion heart muscle cells and destruction of surrounding blood vessels. Once damaged, adult heart cells cannot

Conflict of interest: The author has declared that no conflict of interest exists.

Citation for this article: $J$ Clin Invest. 2014;

124(3):961-964. doi:10.1172/JCI74418. be replaced through regeneration; therefore, an alternate form of wound healing is orchestrated by the immune system. Inflammatory cells migrate to the injured heart, ensuring the clearance of harmful cell debris and repair of the damaged area via formation of a fibrotic scar. The MI-associated inflammatory response is largely stereotypical and considered to be a function of the innate immune system. While inflammatory tissue reparation is initially beneficial, the scarring leads to pathological remodeling of the heart and compromised cardiac function over time. Moreover, if the initial acute inflammatory response becomes chronic, host tissue will be subject to continued damage.

Efforts to promote heart regeneration have largely centered on mechanisms to replace lost cardiovascular cells via cell transplantation or through stimulation of resident cells, such as altering progenitor cell differentiation or inducing muscle cell division. Relatively little attention has been paid to the local environment that transplanted or newly stimulated cells encounter in the heart following MI, which constitutes a proinflammatory milieu, much of which is cytotoxic, and an ensuing fibrosis that disrupts essential matrix. Together, the proinflammatory environment along with a dysfunctional matrix prevent engraftment and integration of new cells into survived heart tissue. While the default immune response to MI appears to ensure a quick fix of the heart, it precludes cell replacement and tissue regeneration. 
A

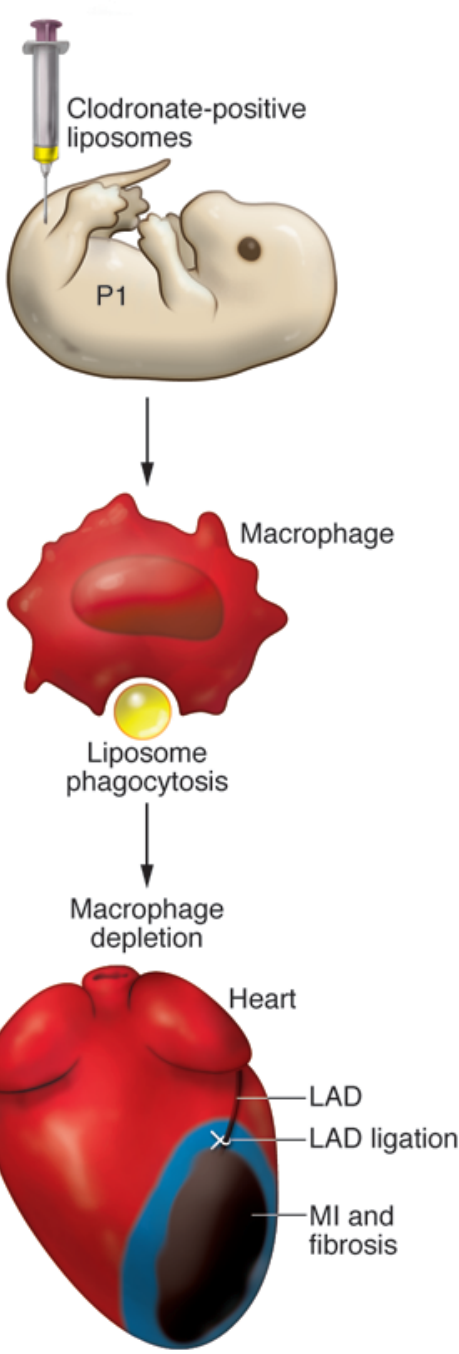

B
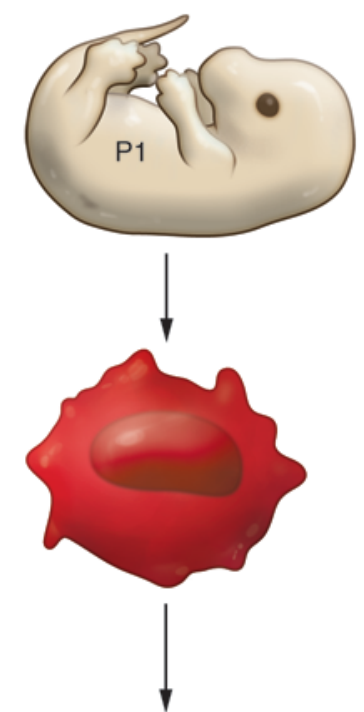

Uniform

macrophage response

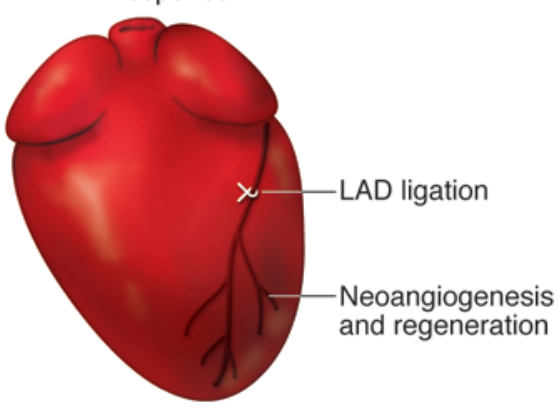

Figure 1

Macrophage depletion prevents regeneration of the neonatal mouse heart. (A) Neonatal mouse pups are intravenously injected with clodronate-loaded liposomes (yellow), which are phagocytosed by macrophages (red). Clodronate uptake results in macrophage apoptosis and subsequent depletion (blue). Loss of macrophages in neonatal mice results in scarring and fibrosis following MI. (B) In untreated pups, macrophages infiltrate the injured heart and are distributed uniformly throughout the heart (red) following MI. Macrophage infiltration into the heart is proposed to stimulate coronary neovascularization, via paracrine secretion of proangiogenic factors, to support myocardial regeneration. LAD, left anterior descending artery.

\section{Macrophage influx in cardiac wound healing and regeneration}

In this issue of JCI, Aurora and colleagues (1) exploited the remarkable ability of the neonatal mouse heart to completely regenerate following injury in the first week of life (2) to study inflammation in heart repair. Aurora et al. focused on monocytes and their activated derivatives, macrophages. In adult animals, monocytes are the dominant cell infiltrate involved in healing injured myocardium within the first two weeks after injury. Monocytes arise from reservoirs

in the bone marrow and spleen, alongside a further subpopulation resident within the tissue itself. When mobilized into the circulation, monocytes patrol blood vessels and respond to chemokines released from the injured tissue to infiltrate and differentiate irreversibly into macrophages (3). Once differentiated, macrophages are capable of engulfing dying cells at the site of injury; however, they release proteolytic enzymes and reactive oxygen species, which harm surviving cells and exacerbate the injury. While it appears that macrophages may do more harm than good in tissue repair, prevention of macrophage infiltration into the injured myocardium negatively correlates with healing and heart function. Seminal work by Nahrendorf and colleagues (4) reconciled this apparent conflict by revealing a biphasic wave of recruitment of subsets of monocytes and macrophages to the injured heart. The biphasic response is comprised of an early influx of proinflammatory monocytes, defined as Ly-6Chi (M1), which promote digestion of infarcted tissue and removal of necrotic debris, followed by infiltration of reparative Ly-6C ${ }^{\text {lo }}$ (M2 or alternatively activated) monocytes, which propagate repair through collagen scar formation and coronary angiogenesis during the resolution of inflammation (3). A dual requirement for both $\mathrm{M} 1$ and M2 monocyte subsets is further emphasized by observations made when monocytes are depleted or elevated. Splenectomized mice, monocyte-depleted mice (4-6), and patients on steroids that reduce monocyte number (7) exhibit impaired wound healing following MI. Patients with elevated monocytosis (8, 9) and apolipoprotein E-null (ApoE-null) mice, which also have enhanced monocyte levels (10) also exhibit reduced MI-associated wound healing. Together, these data suggest that timely resolution of the inflammatory infiltrate and spatial constraint of the reparative response to the site of injury are essential for optimal healing following cardiac injury (11).

It is well established that monocytes and macrophages are essential for repairing MIinduced damage; however, the contribution of these cells in cardiovascular regeneration has not been appreciated. Aurora and colleagues profiled monocyte-derived populations in the hearts and spleens of mice that had undergone MI at P1, an age at which the neonatal heart can regenerate, or MI at P14, an age at which regenerative capacity is lost and replaced by scar formation (1). Aurora et al. observed an overall decrease in heart and splenic macrophages 7 days after MI in P1 mice compared with P14 mice. Furthermore, macrophages localized to the heart at different times, depending on the age of the animal at the time of MI. In P1 mice, macrophages were more abundant and distributed uniformly throughout the heart following MI, while in P14 mice, macrophages were fewer in number and localized almost exclusively to the site of injury (infarct zone) after MI. These data suggest a global macrophage response in P1 mice following injury. In 
adult zebrafish, which are able to regenerate heart tissue, other organ-wide events, including epicardial activation and cardiomyocyte proliferation, are observed during heart repair $(12,13)$. The significance of an organ-wide macrophage response to injury in the murine neonatal heart and the molecular cues that instruct localization to remote myocardium are unclear.

Based on the observed differences in macrophage responses in P1 mice and P14 mice following MI, Aurora and colleagues hypothesized that differences in abundance and localization might reflect a regenerative role for macrophages in mice undergoing MI at P1 (1). The importance of macrophages in P1 mouse heart regeneration was then tested directly by depleting them with clodronate-loaded liposomes prior to MI (14) (Figure 1). Strikingly, macrophage-depleted P1 mice could not regenerate heart tissue following MI and instead developed cardiac scarring accompanied by reduced cardiac function (Figure 1). Evaluation of neutrophils in P1 mice and P14 mice after MI revealed no differences in this immune cell population. Furthermore, despite the phagocytic property of neutrophils, clodronate liposome depletion appeared to be macrophage specific, likely due to the rapid and short-lived neutrophil response (11). It remains to be determined whether neutrophils contribute to heart regeneration.

\section{A unique macrophage population promotes neonatal cardiac regeneration}

Transcriptional profiling revealed striking differences in gene expression between monocyte populations isolated from P1 mice following MI and monocyte populations isolated from P14 mice following MI. Analysis of candidate markers of both M1 (Cd86, Fcgr1, and Nos2) and M2 (chitinaselike 3 [Chi3l3] and arginase 1 [Arg1]) subtypes revealed that there was only a modest increase in candidate marker expression and no clear bias toward an M1 or an M2 response following $\mathrm{MI}$ in $\mathrm{P} 1$ mice; however, all markers were elevated in the monocyte population isolated from $\mathrm{P} 14$ mice after MI, including a substantial increase in the M2 marker Arg1. Subsequent microarray analysis revealed upregulation of transcripts involved in inflammation, angiogenesis, and oxidative stress in macrophages in P1 mice after MI, while following MI in P14 mice, the antiinflammatory factor Il10 was upregulated. Interestingly, monocyte populations from either P1 or P14 mice could not be definitively categorized as M1 or M2, suggesting that the canonical adult monocyte populations are not involved in neonatal responses to MI. Investigation of the cellular effects within the regenerating myocardium revealed that cardiomyocyte proliferation, which underpins myocardial repair (2), was unaffected in P1 mice that had been depleted of macrophages prior to MI; however, neovascularization, which restores blood flow to ischemic and newly formed myocardium, was impaired, suggesting that P1 macrophages promote coronary angiogenesis.

\section{Conclusions and future directions}

A general role for macrophages in tissue regeneration is evident across multiple species. In the axolotl, an aquatic salamander, macrophages are required during the early phase of limb regeneration (15), suggesting an evolutionary conservation of regenerative macrophages from amphibians to mammals. However, since the axolotl is neotenic, an essentially juvenile form, it will be interesting to extrapolate the requirement for macrophages in juvenile tissue regeneration to an adult model of heart regeneration, such as the zebrafish.

A number of important questions remain regarding the role of macrophages in neonatal mouse heart regeneration. Monocytes represent a heterogeneous mix in the adult heart based on immune phenotypes, proinflammatory versus antiinflammatory status, and reparative profiles (16). Aurora and colleagues attempted to stratify the neonatal populations according to the well-described M1 and M2 subtypes, but it is apparent that the macrophage population derived from P1 neonates following MI represents a distinct and unique subset: therefore, this macrophage population likely performs different functions compared with macrophages isolated from P14 and adult mice after injury (1). Due to the distinct characteristics of the regenerative macrophages identified in P1 mice, translating the findings of Aurora and colleagues for targeting monocytes/macrophages during adult heart injury will be complex moving forward. Additionally, the mechanisms used by the $\mathrm{P} 1$ regenerative macrophage subset to promote regeneration are unclear, although a proangiogenesis function was implicated. It is possible that regenerative macrophages act on resident fibroblast and myofibroblast populations, which mediate fibrosis and scarring after MI; however, these interactions have not been explored. Interestingly, recent evidence indicates cross talk between macrophage and stem cell populations (17), which could contribute to neonatal mouse heart regeneration $(2,18)$.

The signals required to reprogram adult macrophages or induce a biphasic recruitment of subtypes into the infarcted adult heart remain unknown. It is also unclear how the transition from the $\mathrm{P} 1$ regenerative phenotype to an immunophenotype is regulated and which injury-induced factors are involved. Interestingly, Aurora and colleagues found that Tregs at P1 were largely undetectable, only emerging after P4 (1). Given that Tregs promote monocyte differentiation toward an antiinflammatory/ reparative profile (19), the lymphocyte response may induce the immuno-switch in macrophages beyond P1.

Aurora and colleagues profiled changes in absolute numbers of heart and splenic macrophages in P1 and P14 mice after MI (1); however, due to the global macrophage depletion by clodronate, the source of regenerative macrophages was not determined. Macrophages that localize to the heart following injury could infiltrate via the circulation from remote sources, such as bone marrow, or progenitors seeded in hemogenic endothelium and yolk sac blood islands or derive from local precursors in the heart (16). Macrophages that originate from sources other than bone marrow and spleen do not necessarily confer inflammatory functions, but in the adult heart, tissue macrophages $\left(\mathrm{CX} 3 \mathrm{CR} 1^{+}\right)$are the predominant form in the myocardium and resemble the alternatively activated antiinflammatory M2 macrophages (20). Whether an equivalent resident population resides in the neonatal heart remains to be determined.

Despite outstanding questions, the finding that macrophages are required for heart regeneration is very compelling and potentially paradigm shifting. Although more work will be required to recapitulate regenerative macrophage function in the adult heart, targeting immunomodulation following MI, along with cell-based regenerative therapies, has potential for optimal cardiovascular repair.

\section{Acknowledgments}

P.R. Riley is a British Heart Foundation Professor of Regenerative Medicine, supported by BHF grants: $\mathrm{CH} / 11 / 1 / 28798$ and RG/13/9/30269. 
Address correspondence to: Paul R. Riley, University of Oxford, Department of Physiology, Anatomy and Genetics, Sherrington Building, South Parks Road, Oxford, OX1 3PT, United Kingdom. Phone: 44.0.1865.282366; Fax: 44.0.1865.272469; E-mail: paul.riley@dpag.ox.ac.uk.

1. Aurora AB, et al. Macrophages are required for neonatal heart regeneration. J Clin Invest. 2014; 124(3):1382-1392.

2. Porrello ER, et al. Transient regenerative potential of the neonatal mouse heart. Science. 2011; 331(6020):1078-1080.

3. Nahrendorf M, Pittet MJ, Swirski FK. Monocytes: protagonists of infarct inflammation and repair after myocardial infarction. Circulation. 2010; 121(22):2437-2445.

4. Nahrendorf $M$, et al. The healing myocardium sequentially mobilizes two monocyte subsets with divergent and complementary functions.J Exp Med. 2007;204(12):3037-3047.

5. Swirski FK, et al. Identification of splenic reservoir monocytes and their deployment to inflammatory sites. Science. 2009;325(5940):612-616
6. van Amerongen MJ, Harmsen MC, van Rooijen N, Petersen AH, van Luyn MJ. Macrophage depletion impairs wound healing and increases left ventricular remodeling after myocardial injury in mice. $A m$ I Pathol. 2007;170(3):818-829.

7. Roberts R, DeMello V, Sobel BE. Deleterious effects of methylprednisolone in patients with myocardial infarction. Circulation. 1976;53(3 suppl):I204-I206.

8 . Tsujioka $\mathrm{H}$, et al. Impact of heterogeneity of human peripheral blood monocyte subsets on myocardial salvage in patients with primary acute myocardial infarction. J Am Coll Cardiol. 2009;54(2):130-138.

9. Maekawa Y, et al. Prognostic significance of peripheral monocytosis after reperfused acute myocardial infarction: a possible role for left ventricular remodeling. J Am Coll Cardiol. 2002;39(2):241-246.

10. Panizzi $\mathrm{P}$, et al. Impaired infarct healing in atherosclerotic mice with Ly-6C(hi) monocytosis. J Am Coll Cardiol. 2010;55(15):1629-1638.

11. Frangogiannis NG. The immune system and cardiac repair. Pharmacol Res. 2008;58(2):88-111.

12. Lepilina A, et al. A dynamic epicardial injury response supports progenitor cell activity during zebrafish heart regeneration. Cell. 2006;127(3):607-619.

13. Itou J, et al. Migration of cardiomyocytes is essential for heart regeneration in zebrafish. Development. 2012;139(22):4133-4142.
14. van Rooijen N, van Nieuwmegen R. Elimination of phagocytic cells in the spleen after intravenous injection of liposome-encapsulated dichloromethylene diphosphonate. An enzyme-histochemical study. Cell Tissue Res. 1984;238(2):355-358.

15. Godwin JW, Pinto AR, Rosenthal NA. Macrophages are required for adult salamander limb regeneration. Proc Natl Acad Sci U S A. 2013;110(23):9415-9420.

16. Nahrendorf M, Swirski FK. Monocyte and macrophage heterogeneity in the heart. Circ Res. 2013; 112(12):1624-1633.

17. Ben-Mordechai T, et al. Macrophage subpopulations are essential for infarct repair with and without stem cell therapy. J Am Coll Cardiol. 2013; 62(20):1890-1901.

18. Jesty SA, et al. c-kit+ precursors support postinfarction myogenesis in the neonatal, but not adult, heart. Proc Natl Acad Sci U S A. 2012;109(33):13380-13385.

19. Tiemessen MM, Jagger AL, Evans HG, van Herwijnen MJ, John S, Taams LS. CD4+CD25+Foxp3+ regulatory $\mathrm{T}$ cells induce alternative activation of human monocytes/macrophages. Proc Natl Acad Sci US A. 2007;104(49):19446-19451.

20. Pinto AR, et al. An abundant tissue macrophage population in the adult murine heart with a distinct alternatively-activated macrophage profile. PLoS One. 2012;7(5):e36814.

\title{
Translational enhancers of EAAT2: therapeutic implications for neurodegenerative disease
}

\author{
Allison S. Limpert and Nicholas D.P. Cosford
}

Cell Death and Survival Networks Program, Sanford-Burnham Medical Research Institute, La Jolla, California, USA.

\begin{abstract}
Glutamate excitotoxicity contributes to the neuronal injury and death associated with many neurodegenerative diseases. The glutamate transporter EAAT2, which is primarily localized on astrocytic processes, facilitates glutamate clearance from synapses, thus preventing neuronal damage. In this issue of the JCI, Kong et al. characterize a compound that upregulates EAAT2 translation, thereby increasing glutamate uptake by glial cells. Furthermore, this strategy for alleviating excitotoxicity was found to be beneficial in mouse models of both amyotrophic lateral sclerosis (ALS) and epilepsy, suggesting that future development in this chemical series may lead to much-needed treatments for these disorders.
\end{abstract}

\section{Current therapies for neurodegenerative diseases offer limited relief}

Current treatments for many neurodegenerative diseases provide only very modest effects on disease progression and symptomology. While neurodegenerative disorders such as Parkinson's disease (PD), Alzheimer's disease (AD), and amyotrophic lateral sclerosis (ALS) affect an ever-growing number of individuals, our ability to

Conflict of interest: The authors have declared that no conflict of interest exists.

Citation for this article: $J$ Clin Invest. 2014;

124(3):964-967. doi:10.1172/JCI74608. treat these fatal illnesses is very limited. For example, ALS leads to the degeneration and death of motor neurons, causing muscle wasting, paralysis, and eventual death of affected individuals; however, the lone FDA-approved treatment currently available for this disorder only extends lifespan by a matter of months (1).

In this issue, Kong et al. propose a therapeutic strategy for the treatment of neurodegenerative disorders that targets the neuronal death and dysfunction caused by glutamate excitotoxicity (2). Damage to neurons caused by excess glutamate contributes to ALS and is a feature of many neurological conditions, including epilepsy, which is a group of disorders characterized by epileptic seizures resulting from aberrant cortical neuronal activity (3). Kong and colleagues characterize a compound, LDN/ OSU-0212320, that upregulates the translation of the glutamate transporter EAAT2 in astrocytes, thus increasing EAAT2 on glial cell surfaces and enhancing glutamate uptake (2). Previous work by this group entailed a small-molecule screen in PAEAAT2 cells, an EAAT2-expressing primary astrocyte line, for compounds that induce translational upregulation of EAAT2 (4). This led to a structure-activity relationship (SAR) study performed on the pyridazinebased series of small molecules identified in the aforementioned screen (5). The characterization of LDN/OSU-0212320 is the culmination of this work, demonstrating the efficacy of a representative compound from the pyridazine series in animal models of neuronal disorders.

Kong et al. demonstrate herein that within 2 hours of treatment, LDN/OSU-0212320 enhanced the localization of EAAT2 mRNA within polyribosome cell fractions, enhanced EAAT2 expression on the plasma 